\title{
Block \& replacement regimen is more cost effective than titration regimen in treating thyrotoxicosis in children, without more harm
}

\author{
MK Anne Kwok*, PT Cheung \\ From 8th APPES Biennial Scientific Meeting \\ Darwin, Australia. 29 October - 1 November 2014
}

\begin{abstract}
Aim
The first line treatment of thyrotoxicosis in our unit is anti-thyroid medication for 2 years - longer if unstable control. Since 2010, we changed the anti-thyroid treatment protocol from block and replacement (B\&R) to titration regimen (TIT), after the Cochrane review showed that $B \& R$ was associated with similar remission rate but more frequent adverse effects than TIT. We aimed at comparing these 2 regimens in our pediatric patients.
\end{abstract}

\section{Methods}

Patients who received TIT from 2010 to 2014 for relapse of thyrotoxicosis and previously had at least 1 full course of B\&R were reviewed. Those who had less than 2 years of TIT, either because they received surgery or radioactive iodine within 2 years, or they have not yet finished the course, were excluded from analysis.

The course of $B \& R$ versus TIT was compared within each individual. For those who had previously multiple courses of $B \& R$, the latest course was used for analysis.

Occurrence of adverse drug side effects, length of the anti-thyroid treatment course, number of clinic visits and thyroid function tests during the course, and frequency of abnormal free thyroxine levels were reviewed and analyzed by paired $t$-test.

\section{Results}

27 patients, who received $B \& R$ previously, were put on TIT for relapse of thyrotoxicosis after 2010. 17 patients were excluded from the analysis as they were on TIT for less than 2 years, 9 of them had surgery or radioactive iodine within 2 years after starting TIT.
Among the 10 patients (4 males, 6 females, age 8-16 years) analysed, no significant drug side effect was experienced during both treatment periods. While on TIT, patients required longer duration of treatment (average $23 \%$ longer, $\mathrm{p}=0.005$ ) with more clinic visits (average 50\% increase, $\mathrm{p}=0.003$ ) and thyroid function tests (average $85 \%$ increase, $\mathrm{p}=0.002$ ).

They needed more frequent clinic visits (average 22\% increase, $\mathrm{p}=0.04$ ) and thyroid function tests (average $50 \%$ increase, $\mathrm{p}=0.007$ ) per year. They had higher number of abnormal free thyroxine levels per year (average $28 \%$ more frequent, $\mathrm{p}=0.023$ ). The percentage of abnormal free thyroxine levels dropped insignificantly by $2.6 \%$ $(\mathrm{p}=0.645)$.

The estimated drug cost for 2 year's B\&R was $~ 397$ HKD (51.2 USD / 54.6 AUD), while 2 years' TIT cost $\sim 177$ HKD (22.8 USD / 24.3 AUD).

\section{Conclusion}

Both B\&R and TIT are safe without significant adverse effect. B\&R seems more cost effective with shorter treatment duration and less frequent clinical and biochemical monitoring, even drug cost is slightly higher.

Published: 28 April 2015

doi:10.1186/1687-9856-2015-S1-P105

Cite this article as: Kwok and Cheung: Block \& replacement regimen is more cost effective than titration regimen in treating thyrotoxicosis in children, without more harm. International Journal of Pediatric

Endocrinology 2015 2015(Suppl 1):P105. 